DOI: $10.17516 / 1997-1397-2020-13-5-519-532$

УДК 517.55

\title{
A Perturbation of the de Rham Complex
}

\author{
Ihsane Malass* \\ Nikolai Tarkhanov ${ }^{\dagger}$ \\ University of Potsdam \\ Potsdam, Germany
}

Received 16.05.2020, received in revised form 07.06.2020, accepted 16.07.2020

\begin{abstract}
We consider a perturbation of the de Rham complex on a compact manifold with boundary. This perturbation goes beyond the framework of complexes, and so cohomology does not apply to it. On the other hand, its curvature is "small", hence there is a natural way to introduce an Euler characteristic and develop a Lefschetz theory for the perturbation. This work is intended as an attempt to develop a cohomology theory for arbitrary sequences of linear mappings.
\end{abstract}

Keywords: De Rham complex, cohomology, Hodge theory, Neumann problem.

Citation: I. Malass, N. Tarkhanov, A Perturbation of the de Rham Complex, J. Sib. Fed. Univ. Math. Phys., 2020, 13(5), 519-532. DOI: 10.17516/1997-1397-2020-13-5-519-532.

\section{Introduction}

De Rham cohomology is a tool belonging both to algebraic topology and to differential topology, capable of expressing basic topological information about smooth manifolds in a form particularly adapted to computation and the concrete representation of cohomology classes. It is a cohomology theory based on the existence of differential forms with prescribed properties. We tracked out its analytic ingredients in [5].

In this paper we consider a perturbation of the de Rham complex on a compact manifold with boundary $\mathcal{X}$ of dimension $n$. That is

$$
0 \longrightarrow \Omega^{0}(\mathcal{X}) \stackrel{d+a}{\longrightarrow} \Omega^{1}(\mathcal{X}) \stackrel{d+a}{\longrightarrow} \ldots \stackrel{d+a}{\longrightarrow} \Omega^{n}(\mathcal{X}) \longrightarrow 0
$$

where $\Omega^{i}(\mathcal{X})$ stands for the space of all differential forms of degree $i$ with $C^{\infty}$ coefficients on $\mathcal{X}$, by $d$ is meant the exterior differentiation of forms, and $a$ is a given $C^{\infty}$ one-form on $\mathcal{X}$. The differential in $(0.1)$ is defined by $(d+a) u=d u+a \wedge u$ for all $u \in \Omega^{i}(\mathcal{X})$ whence

$$
\begin{aligned}
(d+a)^{2} u & =d^{2} u+d a \wedge u-a \wedge d u+a \wedge d u+(a \wedge a) \wedge u= \\
& =d a \wedge u
\end{aligned}
$$

The differential square is sometimes referred to as the curvature of sequence $(0.1)$ and it is "small" in some relevant sense. This enables one to introduce the Euler characteristic and prove a Lefschetz fixed point formula for $(0.1)$, see [11,13].

However, $(d+a)^{2}$ need not be zero and for this reason the standard cohomology construction does not apply to (0.1). To introduce cohomology in $(0.1)$ we use a construction of mathematical

\footnotetext{
*ihsane malass@hotmail.com

†tarkhanov@math.uni-potsdam.de

(C) Siberian Federal University. All rights reserved
} 
folklore. To wit, we factorise the null-space $\mathcal{Z}^{i}$ of $d+a$ in $\Omega^{i}(\mathcal{X})$ by the (part of the) range of $d+a$ over $\Omega^{i-1}(\mathcal{X})$ belonging to the space $\mathcal{Z}^{i}$. Thus, we define

$$
H^{i}(\Omega(\mathcal{X}), d+a):=\frac{\mathcal{Z}^{i}}{(d+a)\left(\Omega^{i-1}(\mathcal{X})\right) \cap \mathcal{Z}^{i}}
$$

for $i=0,1, \ldots, n$.

If $f=(d+a) u$ belongs to $\mathcal{Z}^{i}$, then $(d+a) f=(d+a)^{2} u=0$, i.e., $\omega \wedge u=0$ in $\mathcal{X}$, where $\omega=d a$ is a given $C^{\infty}$ two-form on $\mathcal{X}$. Denote by $\Omega_{\omega}^{i}(\mathcal{X})$ the subspace of $\Omega^{i}(\mathcal{X})$ consisting of all forms $u \in \Omega^{i}(\mathcal{X})$ which satisfy $\omega \wedge u=0$ in $\mathcal{X}$. By the very definition, $\Omega_{\omega}^{i}(\mathcal{X})$ coincides with $\Omega^{i}(\mathcal{X})$ for $i=n-1, n$. Given any $u \in \Omega_{\omega}^{i}(\mathcal{X})$, we get

$$
\begin{aligned}
\omega \wedge(d+a) u & =\omega \wedge d u+a \wedge(\omega \wedge u)= \\
& =(d+a)(\omega \wedge u)=0,
\end{aligned}
$$

for $\omega$ is a closed two-form on $\mathcal{X}$. Hence it follows that $d+a$ maps $\Omega_{\omega}^{i}(\mathcal{X})$ into $\Omega_{\omega}^{i+1}(\mathcal{X})$ for all $i=0,1, \ldots, n-1$. We have thus associated the complex of linear mappings

$$
0 \longrightarrow \Omega_{\omega}^{0}(\mathcal{X}) \stackrel{d+a}{\longrightarrow} \Omega_{\omega}^{1}(\mathcal{X}) \stackrel{d+a}{\longrightarrow} \ldots \stackrel{d+a}{\longrightarrow} \Omega_{\omega}^{n}(\mathcal{X}) \longrightarrow 0
$$

to sequence $(0.1)$.

Complex (0.2) gains in interest if we realise that its cohomology just amounts to $H^{i}(\Omega \cdot(\mathcal{X}), d+a)$ at each step $i$.

If the differential form $a$ is closed in all of $\mathcal{X}$, then (0.1) is actually a complex and both (0.1) and $(0.2)$ coincide. Otherwise $\Omega_{\omega}^{i}(\mathcal{X})$ need not be a space of sections of some vector bundle over $\mathcal{X}$. Indeed, the forms $u$ of $\Omega_{\omega}^{i}(\mathcal{X})$ are described by the equation $\omega \wedge u=0$ on $\mathcal{X}$. At any fixed point $x \in \mathcal{X}$ the equation reduces to a system of $C_{n}^{i+2}$ linear equations for $C_{n}^{i}$ unknowns which are the coefficients of $u(x)$. The dimension of the space of solutions to this system just amounts to $C_{n}^{i}$ minus the rank of the bundle homomorphism $\Lambda^{i} T^{*} \mathcal{X} \rightarrow \Lambda^{i+2} T^{*} \mathcal{X}$ determined by the exterior multiplication with $\omega(x)=d a(x)$. Hence, the dimension varies with $x$ and the family of subspaces of $\Lambda^{i} T^{*} \mathcal{X}$ defined pointwise by $\omega \wedge u=0$ fails to constitute a subbundle of $\Lambda^{i} T^{*} \mathcal{X}$ in general. However, if the rank of the bundle homomorphism $d a(x) \wedge$ is constant, i.e., independent of $x \in \mathcal{X}$, then $\Omega_{\omega}^{i}(\mathcal{X})$ is specified within the framework of smooth sections of a subbundle $V^{i}$ of $\Lambda^{i} T^{*} \mathcal{X}$. In this case (0.2) is a complex of first order differential operators between sections of vector bundles over $\mathcal{X}$. However, it is not elliptic, and so the Neumann problem after Spencer [9] does not apply to study the cohomology of complex (0.2).

Example 0.1. If $a$ is an one-form on an open set $U \in \mathbb{R}^{3}$ with non-vanishing coefficient $\omega_{1,2}$ of $\omega=d a$, then $(0.2)$ reduces to

$$
0 \longrightarrow 0 \longrightarrow C^{\infty}\left(U, \mathbb{R}^{2}\right) \stackrel{A^{1}}{\longrightarrow} C^{\infty}\left(U, \mathbb{R}^{3}\right) \stackrel{A^{2}}{\longrightarrow} C^{\infty}(U) \longrightarrow 0
$$

the principal symbols of $A^{1}$ and $A^{2}$ being

$$
\begin{aligned}
\sigma^{1}\left(A^{1}\right)(x, \xi) & =\left(\begin{array}{cc}
q_{1}(x) \xi_{2} & q_{2}(x) \xi_{2}-\xi_{3} \\
-q_{1}(x) \xi_{1}+\xi_{3} & -q_{2}(x) \xi_{1} \\
-\xi_{2} & \xi_{1},
\end{array}\right), \\
\sigma^{1}\left(A^{2}\right)(x, \xi) & =\left(\xi_{1}, \xi_{2}, \xi_{3}\right)
\end{aligned}
$$

up to the factor $\imath=\sqrt{-1}$. Here,

$$
q_{1}=-\frac{\omega_{2,3}}{\omega_{1,2}}, \quad q_{2}=\frac{\omega_{1,3}}{\omega_{1,2}} .
$$

The rank of $\sigma^{1}\left(A^{1}\right)(x, \xi)$ is equal to 1 for all $\xi$ on the line $\xi_{1}=\xi_{2}, \xi_{3}=\left(q_{1}+q_{2}\right) \xi_{2}$ in $\mathbb{R}^{3}$. 
Locally any closed differential form $a$ is exact. If $a=d p$ for some smooth function $p$ on $\mathcal{X}$, then an easy calculation shows that $(d+a) u=e^{-p} d\left(e^{p} u\right)$ for all forms $u \in \Omega^{i}(\mathcal{X})$. In this particular case complex $(0.2)$ is obtained by a similarity transformation from the de Rham complex. This conjugation was used in the work [15] who proved Morse inequality using some spectral information of the Laplacian of the complex. The approach of [4] to solving the $\bar{\partial}$ problem is basically the same, and it is from 1965, predating [15] by a couple of decades. By varying the "weight" function $p$ one can get $L^{2}$ estimates for the solution of $\bar{\partial}$ problem. The paper [4] does not make explicit that it is perturbing the Dolbeault complex, but that is exactly what it is doing. For a further development of this approach to Morse theory we refer the reader to $[6]$ and the references given there.

In this work we focus on the cohomology of complex (0.2) in the case where the form $a$ is not closed. Our basic assumption is that the rank of the bundle homomorphism $d a(x) \wedge$ is constant.

\section{The cohomology of the associated complex}

A complex is said to be Fredholm if its cohomology is finite-dimensional at each step. This concept can be extended within the framework of nonstandard cohomology to arbitrary sequences (0.1). However, it should be noted that it differs from the concept of Fredholm quasicomplexes studied in [11]. Since (0.1) is a "small" perturbation of the de Rham complex on $\mathcal{X}$, it is a Fredholm quasicomplex in the sense of [11]. This allows one to introduce an Euler characteristic of (0.1). Still, the paper [11] does not contain any definition of cohomology for Fredholm quasicomplexes.

Lemma 1.1. As defined above, the cohomology of complex (0.2) at step $i$ coincides with $H^{i}(\Omega \cdot(\mathcal{X}), d+a)$.

Proof. For any $f \in \Omega_{\omega}^{i}(\mathcal{X}) \cap \mathcal{Z}^{i}$, we write $[f]$ for the equivalence class of $f$ in $H^{i}\left(\Omega_{\omega}^{i}(\mathcal{X})\right)$. Let $\iota$ be the embedding of $\Omega_{\omega}^{i}(\mathcal{X}) \cap \mathcal{Z}^{i}$ into $\Omega^{i}(\mathcal{X}) \cap \mathcal{Z}^{i}$. Consider the mapping

$$
\iota_{*}: H^{i}\left(\Omega_{\omega}^{i}(\mathcal{X})\right) \rightarrow H^{i}\left(\Omega_{\omega}^{i}(\mathcal{X}), d+a\right)
$$

which assigns to any class $[f] \in H^{i}\left(\Omega_{\omega}^{i}(\mathcal{X})\right)$ the class of $\iota(f)$ in $H^{i}\left(\Omega^{\cdot}(\mathcal{X}), d+a\right)$. This definition is correct, for if $f=(d+a) u$ with some form $u \in \Omega_{\omega}^{i-1}(\mathcal{X})$, then $(d+a) f=(d+a)^{2} u=\omega \wedge u=0$, i.e., the class of $(d+a) f$ in $H^{i}(\Omega \cdot(\mathcal{X}), d+a)$ is zero.

The mappng $\iota_{*}$ ist injective. For let $f \in \Omega_{\omega}^{i}(\mathcal{X}) \cap \mathcal{Z}^{i}$ satisfy $\iota_{*}[f]=0$. Then there is a form $u \in \Omega^{i-1}(\mathcal{X})$ such that $f=(d+a) u$. Hence it follows immediately that

$$
\begin{aligned}
\omega \wedge u & =(d+a)^{2} u= \\
& =(d+a) f=0
\end{aligned}
$$

i.e., $[f]=0$, as desired.

It remains to show that $\iota_{*}$ ist surjective. To this end, pick an arbitrary form $f \in \mathcal{Z}^{i}$. Then $\omega \wedge f=(d+a)^{2} f=0$, and so $f \in \Omega_{\omega}^{i}(\mathcal{X})$ and $\iota_{*}[f]$ is the class of $f$ in $H^{i}(\Omega \cdot(\mathcal{X}), d+a)$.

The sequence of symbol mappings in (0.1) is exact away from the zero section of $T^{*} \mathcal{X}$, and so $(0.1)$ is an elliptic sequence in the interior of $\mathcal{X}$. Moreover, the sequence of boundary symbols is exact away from the zero section of $T^{*} \partial \mathcal{X}$, both conditions are usually referred to as ellipticity on a manifold with boundary. However, they characterise the Fredholm property in Sobolev spaces while we go beyond these spaces in (0.2). 


\section{Perturbations on 2-dimensional manifolds}

To learn complex (0.2) we consider the particular case $n=2$, i.e., $\mathcal{X}$ is a manifold of dimension 2 .

If $d a\left(x_{0}\right) \neq 0$ at some point $x_{0} \in \mathcal{X}$, then by continuity $d a(x) \neq 0$ holds for all $x$ in a neighbourhood $U$ of $x_{0}$ on $\mathcal{X}$. We restrict our attention to some local chart $U$ in $\mathcal{X}$ with this property.

Write $a=a_{1} d x^{1}+a_{2} d x^{2}$ in the coordinates of $U$, then $d a=\left(\partial_{1} a_{2}-\partial_{2} a_{1}\right) d x^{1} \wedge d x^{2}$ is different from zero in $U$. By definition, we get

$$
\Omega_{\omega}^{0}(U)=\left\{u \in C^{\infty}(U):\left(\partial_{1} a_{2}-\partial_{2} a_{1}\right) u d x^{1} \wedge d x^{2}=0\right\}=\{0\}
$$

and $\Omega_{\omega}^{i}(U)=\Omega^{i}(U)$ for $i=1,2$ since $d a \wedge u$ has degree at least 3, if $u \in \Omega^{i}(U)$. Complex $(0.2)$ thus becomes

$$
0 \longrightarrow 0 \longrightarrow \Omega^{1}(U) \stackrel{d+a}{\longrightarrow} \Omega^{2}(U) \longrightarrow 0 .
$$

The symbol sequence of (2.1) over a point $(x, \xi) \in T^{*} U$ reduces immediately to the complex

$$
0 \longrightarrow 0 \longrightarrow \Lambda^{1} T_{x}^{*} U \stackrel{\xi \wedge}{\longrightarrow} \Lambda^{2} T_{x}^{*} U \longrightarrow 0
$$

which is not exact at the term $\Lambda^{1} T_{x}^{*} U$, for $\xi \wedge \xi=0$ for all $\xi \in T_{x}^{*} U$. The exactness at the term $\Lambda^{2} T_{x}^{*} U$ is well known for all $\xi \in \mathbb{R}^{2} \backslash\{0\}$. It follows that complex (2.1) fails to be elliptic.

We now turn to the cohomology of (2.1). Namely, given any form $f \in \Omega^{2}(U)$, we look for a solution $u \in \Omega^{1}(U)$ to the inhomogeneous equation $(d+a) u=f$ in $U$. On writing

$$
\begin{aligned}
u & =u_{1} d x^{1}+u_{2} d x^{2}, \\
f & =f_{12} d x^{1} \wedge d x^{2}
\end{aligned}
$$

we reduce the equation to

$$
\left(\partial_{1}+a_{1}\right) u_{2}-\left(\partial_{2}+a_{2}\right) u_{1}=f_{12}
$$

in $U$.

An important class of solutions to this equation is constituted by the so-called potential solutions, i.e., those of the form $u=d p$ where $p$ is a smooth function in $U$ satisfying $a \wedge d p=f$, i.e.,

$$
a_{1} \partial_{2} p-a_{2} \partial_{1} p=f_{12} .
$$

This first order partial differential equation is known to have a unique solution $p$ with prescribed data on any curve $\mathcal{S}$ in $U$ which is not characteristic, i.e., the vector field $\left(-a_{2}, a_{1}\right)$ is tangent to $\mathcal{S}$ at no point. Hence it follows that the cohomology of complex (2.1) is infinite dimensional at the term $\Omega^{1}(U)$ and zero at the term $\Omega^{2}(U)$.

Thus, sequence (0.1) fails to be Fredholm in the sense of Section 1. unless the form $a(x)$ is closed. A substantial theory is hardly expected for the case of nonclosed differential forms $a$, cf. [6]. If $a$ is a closed one-form, then complex (0.1) falls into a useful general construction in homological algebra called the Koszul complex, see Section 1.2.8 of [10].

\section{Quasicomplexes}

In this section we recall some basic facts about complexes and quasicomplexes in Hilbert spaces. For the theory of quasicomplexes of Banach spaces we refer to [2] where quasicomplexes are called essential complexes. 
Let us consider the sequence

$$
\left(H^{\cdot}, d\right): 0 \longrightarrow H^{0} \stackrel{d^{0}}{\longrightarrow} H^{1} \stackrel{d^{1}}{\longrightarrow} \ldots \stackrel{d^{N-1}}{\longrightarrow} H^{N} \longrightarrow 0
$$

where $H^{i}$ are Hilbert spaces and $d^{i}$ are continuous linear maps. The sequence $\left(H^{*}, d\right)$ is called a complex if $d^{i} d^{i-1}=0$ for all $i=1, \ldots, N$. The elements of the spaces $Z^{i}\left(H^{*}, d\right)=\operatorname{ker} d^{i}$ and $B^{i}\left(H^{\prime}, d\right)=\mathrm{im} d^{i-1}$ are called cocycles and coboundaries, respectively. The quotient space $H^{i}\left(H^{*}, d\right)=\operatorname{ker} d^{i} / \operatorname{im} d^{i-1}$ is the cohomology of the complex $\left(H^{*}, d\right)$ at step $i$. The complex $\left(H^{*}, d\right)$ is said to be Fredholm if its cohomology is finite dimensional at each step $i=0, \ldots, N$.

It is well known that "small" perturbations of Fredholm operators do not affect the Fredholm property. For example, perturbing a single Fredholm operator by a compact operator gives us a Fredholm operator. It would be natural to have the same property for Fredholm complexes. However, a "small" perturbation of a Fredholm complex need not be even a complex anymore, i.e., the operators no longer satisfy $d^{i} d^{i-1}=0$.

Note that perturbing an elliptic complex by lower order terms does not change the complex of principal symbols which remains to be exact. Hence, instead of complexes it is natural to consider sequences $\left(H^{*}, d\right)$ with the property that the compositions $d^{i} d^{i-1}$ are "small" in some sense. By "small" operators one usually means compact operators. Let us denote by $\mathcal{K}(H, \tilde{H})$ the subspace of $\mathcal{L}(H, \tilde{H})$ consisting of compact operators.

Definition 3.1. A sequence $\left(H^{*}, d\right)$ of Hilbert spaces $H^{i}$ and continuous linear maps $d^{i}$ is a quasicomplex if $d^{i} d^{i-1} \in \mathcal{K}\left(H^{i-1}, H^{i+1}\right)$ for all $i=1, \ldots, N$.

For $d^{1}, d^{2} \in \mathcal{L}(H, \tilde{H})$, we write $d^{1} \sim d^{2}$ if $d^{1}-d^{2} \in \mathcal{K}(H, \tilde{H})$. It is known that an operator $d \in \mathcal{L}(H, \tilde{H})$ is Fredholm if and only if its image in the Calkin algebra $\mathcal{L}(H, \tilde{H}) / \mathcal{K}(H, \tilde{H})$ is invertible. Hence, the idea of Fredholm quasicomplexes is to pass in a given quasicomplex to quotients modulo spaces of compact operators and require exactness. To make the definition precise we introduce a functor $\phi_{\Sigma}$ studied in [7].

Taking an arbitrary Hilbert space $\Sigma$, we set $\phi_{\Sigma}\left(H^{i}\right)=\mathcal{L}\left(\Sigma, H^{i}\right) / \mathcal{K}\left(\Sigma, H^{i}\right)$ for each Hilbert space $H^{i}$. Then, for any map $d^{i} \in \mathcal{L}\left(H^{i}, H^{i+1}\right)$, we introduce a map $\phi_{\Sigma}\left(d^{i}\right) \in$ $\mathcal{L}\left(\phi_{\Sigma}\left(H^{i}\right), \phi_{\Sigma}\left(H^{i+1}\right)\right)$ by

$$
\phi_{\Sigma}\left(d^{i}\right)\left(A+\mathcal{K}\left(\Sigma, H^{i}\right)\right)=d^{i} A+\mathcal{K}\left(\Sigma, H^{i+1}\right)
$$

for all $A \in \mathcal{L}\left(\Sigma, H^{i}\right)$. Obviously, this operator is well defined. It is easily seen that $\phi_{\Sigma}\left(d^{i} d^{i-1}\right)=$ $=\phi_{\Sigma}\left(d^{i}\right) \phi_{\Sigma}\left(d^{i-1}\right)$ and that $\phi_{\Sigma}$ vanishes on compact operators for every Hilbert space $\Sigma$. Hence, if $\left(H^{*}, d\right)$ is a quasicomplex then $\left(\phi_{\Sigma}\left(H^{\cdot}\right), \phi_{\Sigma}(d)\right)$ is a complex for each Hilbert space $\Sigma$.

Definition 3.2. A quasicomplex $\left(H^{*}, d\right)$ is Fredholm if the complex $\left(\phi_{\Sigma}\left(H^{\cdot}\right), \phi_{\Sigma}(d)\right)$ is exact for each Hilbert space $\Sigma$.

Let $\left(H^{*}, d\right)$ and $\left(H^{*}, \tilde{d}\right)$ be two quasicomplexes of Hilbert spaces, such that $d^{i} \sim \tilde{d}^{i}$ for any $i=0,1, \ldots, N$. Then the complexes $\left(\phi_{\Sigma}\left(H^{*}\right), \phi_{\Sigma}(d)\right)$ and $\left(\phi_{\Sigma}\left(H^{*}\right), \phi_{\Sigma}(\tilde{d})\right)$ coincide. Hence, the quasicomplexes $\left(H^{*}, d\right)$ and $\left(H^{*}, \tilde{d}\right)$ are Fredholm simultaneously. Thus, any compact perturbation of a Fredholm quasicomplex is a Fredholm quasicomplex.

A sequence

$$
(H, \pi): 0 \longleftarrow H^{0} \stackrel{\pi^{1}}{\longleftarrow} H^{1} \stackrel{\pi^{2}}{\longleftarrow} \ldots \stackrel{\pi^{N}}{\longleftarrow} H^{N} \longleftarrow 0
$$

with $\pi^{i} \in \mathcal{L}\left(H^{i}, H^{i-1}\right)$ is said to be a parametrix of the quasicomplex $\left(H^{*}, d\right)$, provided

$$
\pi^{i+1} d^{i}+d^{i-1} \pi^{i}=I_{H^{i}}-\varkappa^{i}
$$

for all $i=0,1, \ldots, N$, where $\varkappa^{i} \in \mathcal{K}\left(H^{i}\right)$.

It is well known that a complex of Hilbert spaces is Fredholm if and only if it has a parametrix. The same property is also true for quasicomplexes, see [11]. 
Theorem 3.3. A quasicomplex $\left(H^{*}, d\right)$ is Fredholm if and only if it possesses a parametrix.

Obviously, if a parametrix $\left(H^{*}, \pi\right)$ of a quasicomplex $\left(H^{*}, d\right)$ is a quasicomplex itself, then $\left(H^{\cdot}, d\right)$ is in turn a parametrix of $\left(H^{*}, \pi\right)$.

It should be noted that Theorem 3.3 does not extend to arbitrary complexes of Banach spaces. The advantage of using Hilbert spaces lies in the fact that any quasicomplex of Hilbert spaces admits the so-called adjoint quasicomplex. By this is meant

$$
\left(H^{*}, d^{*}\right): 0 \longleftarrow H^{0} \stackrel{d^{0 *}}{\longleftarrow} H^{1} \stackrel{d^{1 *}}{\longleftarrow} \ldots \stackrel{d^{N-1 *}}{\longleftarrow} H^{N} \longleftarrow 0,
$$

where $d^{i *} \in \mathcal{L}\left(H^{i+1}, H^{i}\right)$ stands for the adjoint of $d^{i}$ in the sense of Hilbert spaces. Since $d^{i *} d^{i+1 *}=\left(d^{i+1} d^{i}\right)^{*}$ are compact operators, $\left(H^{*}, d^{*}\right)$ is a quasicomplex indeed. The selfadjoint operators $\Delta^{i}=d^{i-1} d^{i-1 *}+d^{i *} d^{i}$ are called the Laplacians of the quasicomplex $\left(H^{*}, d\right)$. The null-space of $\Delta^{i}$ consists of all $h \in H^{i}$ satisfying $d^{i} h=0$ and $d^{i-1 *} h=0$, as is easy to see.

Theorem 3.4. A quasicomplex $\left(H^{*}, d\right)$ is Fredholm if and only if all its Laplacians $\Delta^{i}$ are Fredholm operators.

Proof. See Lemma 4.2 of [12].

As is proved in [11], every Fredholm quasicomplex can actually be transformed into a complex. Another way of stating this theorem is to say that each Fredholm quasicomplex is a perturbation of a Fredholm complex by compact operators.

Theorem 3.5. For any Fredholm quasicomplex $\left(H^{\cdot}, d\right)$ there are operators $D^{i} \in \mathcal{L}\left(H^{i}, H^{i+1}\right)$ satisfying $D^{i} \sim d^{i}$ and $D^{i} D^{i-1}=0$ for all $i$.

\section{A parametrix of the perturbation}

We now return to the perturbation of the de Rham complex on $\mathcal{X}$ defined in (0.1). In order to rewrite it in the context of Hilbert spaces, we choose any integer number $s \geqslant n$ and set $s_{i}=s-i$ for $i=0,1, \ldots, n$. Consider the sequence of linear mappings

$$
0 \longrightarrow H^{s_{0}}\left(\mathcal{X}, \Lambda^{0}\right) \stackrel{d+a}{\longrightarrow} H^{s_{1}}\left(\mathcal{X}, \Lambda^{1}\right) \stackrel{d+a}{\longrightarrow} \ldots \stackrel{d+a}{\longrightarrow} H^{s_{n}}\left(\mathcal{X}, \Lambda^{n}\right) \longrightarrow 0,
$$

where $\Lambda^{i}=\Lambda^{i} T^{*} \mathcal{X}$ is the bundle of exterior forms of degree $i$ over $\mathcal{X}$ and by $H^{s_{i}}\left(\mathcal{X}, \Lambda^{i}\right)$ is meant the space of all differential forms of degree $i$ with coefficients of the Sobolev class $H^{s_{i}}=W^{s_{i}, 2}$ on $\mathcal{X}$. We fix a unitary structure in each of these spaces, thus obtaining a sequence of Hilbert spaces and their continuous linear mappings. By the above, the curvature of (4.1) just amounts to the bundle homomorphism of $\Lambda^{i-1}$ to $\Lambda^{i+1}$ defined via the exterior multiplication by the two-form $d a$. On applying the Rellich theorem we conclude readily that it is a compact operator from $H^{s_{i-1}}\left(\mathcal{X}, \Lambda^{i-1}\right)$ to $H^{s_{i+1}}\left(\mathcal{X}, \Lambda^{i+1}\right)$ for all $i=1, \ldots, n$. Hence, (4.1) is a quasicomplex.

We next show that this quasicomplex is Fredholm. By Theorem 3.3, for this purpose it suffices to construct a parametrix of (4.1). To this end, we use the parametrix of the de Rham complex on $\mathcal{X}$ obtained from the Neumann problem after Spencer, see Section 4 of [5]. More precisely, there are operators $G^{i}$ of order -2 in Boutet de Monvel's algebra of pseudodifferential operators acting in $\Omega^{i}(X)$, such that

$$
f=H f+\left(d^{*} G\right) d f+d\left(d^{*} G\right) f
$$

for all $f \in \Omega^{i}(\mathcal{X})$. Here, $H$ stands for the orthogonal projection in $L^{2}\left(\mathcal{X}, \Lambda^{i}\right)$ onto the finitedimensional subspace of harmonic forms, i.e., those $h \in \Omega^{i}(\mathcal{X})$ which satisfy $d h=0, d^{*} h=0$ in $\mathcal{X}$ and $n(f)=0$ on the boundary of $\mathcal{X}$, where $n(f)$ is the normal part of $f$ on $\partial \mathcal{X}$. By $d^{*}$ is meant the formal adjoint for the exterior derivative with respect to the $L^{2}$ scalar product in 
$\Omega^{i}(\mathcal{X})$. The operator $G^{i}$ satisfies $n(G f)=0$ and $n(d G f)=0$ on the boundary of $\mathcal{X}$ for any $f \in \Omega^{i}(\mathcal{X})$. It is usually referred to as the Green operator of the Hodge theory on manifolds with boundary. Thus, on sufficiently smooth forms $P=d^{*} G$ is a very special parametrix of the de Rham complex.

Lemma 4.1. Let $s$ be an arbitrary nonnegative integer. As defined above, the Green operator $G$ extends to a continuous mapping of $H^{s}\left(\mathcal{X}, \Lambda^{i}\right)$ into $H^{s+2}\left(\mathcal{X}, \Lambda^{i}\right)$.

Proof. Since $u=G f$ gives a solution to the Neumann problem after Spencer for the de Rham complex on $\mathcal{X}$ and this latter problem is elliptic, the desired assertion follows from the regularity theorem for elliptic boundary value problems in Sobolev spaces, see [1] and elsewhere.

The Rellich theorem on compact embeddings of Sobolev spaces implies that sequence (4.1) is a compact perturbation of the de Rham complex evaluated in Sobolev spaces. The shortest way to derive the Fredholm property of (4.1) from here is given by the next theorem.

Theorem 4.2. The sequence of pseudodifferential operators $P^{i}=d^{*} G^{i}$ of order -1 defines a parametrix of sequence (4.1).

Proof. Using equality (4.2), we get

$$
\begin{aligned}
P^{i+1}(d+a) f+(d+a) P^{i} f & =\left(P^{i+1} d f+d P^{i} f\right)+\left(P^{i+1}(a \wedge f)+a \wedge P^{i} f\right)= \\
& =f-K^{i} f
\end{aligned}
$$

for all $f \in H^{s_{i}}\left(\mathcal{X}, \Lambda^{i}\right)$, where

$$
K^{i} f=H^{i} f-\left(P^{i+1}(a \wedge f)+a \wedge P^{i} f\right) .
$$

The projector $H^{i}$ is an operator with smooth Schwartz kernel on the product $\mathcal{X} \times \mathcal{X}$, and so it is a compact operator on $H^{s_{i}}\left(\mathcal{X}, \Lambda^{i}\right)$. On the other hand, the operator $P^{i+1}(a \wedge \cdot)+a \wedge P^{i}$. acts on $H^{s_{i}}\left(\mathcal{X}, \Lambda^{i}\right)$ through the compact embedding $H^{s_{i}+1}\left(\mathcal{X}, \Lambda^{i}\right) \hookrightarrow H^{s_{i}}\left(\mathcal{X}, \Lambda^{i}\right)$, which is due to the Rellich theorem. Hence, this operator is compact as well. On summing up we conclude that $K^{i} \in \mathcal{K}\left(H^{s_{i}}\left(\mathcal{X}, \Lambda^{i}\right)\right)$, as desired.

On applying the paper [11] we are in a position to introduce the Euler characteristic of Fredholm quasicomplex (4.1). To wit, $\chi\left(H^{s \cdot}\left(\mathcal{X}, \Lambda^{*}\right), d+a\right)$ is defined to be equal to the Euler characteristic $\chi(\mathcal{X})$ of $\mathcal{X}$, i.e., to that of the de Rham complex on $\mathcal{X}$.

Our next goal is to improve the parametrix $P^{i}=d^{*} G^{i}$ of quasicomplex (4.1). It is surprising that the standard procedure using the formal Neumann series for $\left(I-K^{i}\right)^{-1}$ no longer works to do this modulo smoothing operators. By abuse of notation we omit the indices of $P^{i}, K^{i}$, etc., thus using the graded operators $P$ and $K$ defined by $P f=P^{i} f$ and $K f=K^{i} f$ for $f \in \Omega^{i}(\mathcal{X})$, respectively. According to (4.3) we get

$$
K=-P a-a P
$$

modulo the harmonic projection.

Lemma 4.3. For any $k=0,1, \ldots$, the commutator $\left[K^{k}, d+a\right]:=K^{k}(d+a)-(d+a) K^{k}$ just amounts to

$$
\left[K^{k}, d+a\right]=-\sum_{j=0}^{k-1} K^{j}[P,(d a)] K^{k-1-j} .
$$

Recall that by $a$ is meant the operator on differential forms given by $f \mapsto a \wedge f$. In contrast to the composition $d a=d \circ a$ we write $(d a)$ for the operator defined by the differential of $a$. 
Proof. For $k=0$ the assertion is obvious, so we start with $k=1$. We first observe that

$$
\begin{aligned}
{[K, d] } & =[H-P a-a P, d]= \\
& =-[P a+a P, d],
\end{aligned}
$$

for $[H, d]=0-0$ is zero by the very definition of harmonic projection $H$. Hence it follows that

$$
\begin{aligned}
{[K, d] } & =d P a+(d a) \wedge P-a d P-P a d-a P d= \\
& =(d P+P d) a-P d a-P a d+(d a) \wedge P-a(d P+P d)= \\
& =(I-H) a-[P,(d a)]-a(I-H)= \\
& =-[H, a]-[P,(d a)]
\end{aligned}
$$

the commutator $[H, a]$ being smoothing and of finite rank. On the other hand, we get

$$
[K, a]=(H-P a-a P) a-a(H-P a-a P)=[H, a],
$$

for $a \wedge a=0$. Thus, $[K, d+a]=-[P,(d a)]$, as desired.

For arbitrary integer $k>1$ we proceed successively using the equality for $k=1$. To wit,

$$
\begin{aligned}
{\left[K^{k}, d+a\right] } & =K^{k-1} K(d+a)-(d+a) K^{k}= \\
& =K^{k-1}(d+a) K-K^{k-1}[P,(d a)]-(d+a) K^{k}= \\
& =K^{k-2}(d+a) K^{2}-K^{k-2}[P,(d a)] K-K^{k-1}[P,(d a)]-(d+a) K^{k},
\end{aligned}
$$

etc., which proves (4.4).

Note that each summand on the right-hand side of (4.4) is a pseudodifferential operator of order $-k$.

Theorem 4.4. Given any $N=0,1, \ldots$, the operators $P_{N}^{i}=\left(\sum_{k=0}^{N}\left(K^{i-1}\right)^{k}\right) P^{i}$ satisfy

$$
P_{N}(d+a)+(d+a) P_{N}=I-K^{N+1}+\sum_{k=0}^{N}\left(\sum_{j=0}^{k-1} K^{j}[P,(d a)] K^{k-1-j}\right) P .
$$

Proof. On multiplying both sides of (4.3) by $\sum_{k=0}^{N} K^{k}$ from the left we immediately obtain

$$
P_{N}(d+a)+(d+a) P_{N}=I-K^{N+1}-\left[\sum_{k=0}^{N} K^{k}, d+a\right] P .
$$

Substituting the expressions for $\left[K^{k}, d+a\right]$ of (4.4) into the latter equality yields the desired formula.

In particular, if the differential form $a$ is closed, then the operators $P_{N}^{i}$ constitute a parametrix of complex (4.1) up to a remainder of order $-N-1$, more precisely, $K^{N+1}$. For perturbations of nonzero curvature there is an additional residual term depending linearly on $d a$.

\section{Local calculation of the Laplacian}

Denote by $\Delta_{a}=(d+a)^{*}(d+a)+(d+a)(d+a)^{*}$ the Laplacian of the perturbed complex. A trivial verification shows that

$$
\Delta_{a}=\Delta_{0}+\left(d^{*} a+a^{*} d+d a^{*}+a d^{*}\right)+\left(a^{*} a+a a^{*}\right),
$$


where $\Delta_{0}$ is the Laplacian of the de Rham complex on $\mathcal{X}$. From this equality we deduce immediately that $\Delta_{a}$ is an elliptic operator of order two at each step $i=0,1, \ldots, n$.

The Laplacian $\Delta_{a}$ has especially simple form $\Delta_{0}+\left(a^{*} a+a a^{*}\right)$ if the first order differential operator $d^{*} a+a^{*} d+d a^{*}+a d^{*}$ vanishes. This latter is the case if and only if the one-form $a$ satisfies an overdetermined system of first order partial differential equations on $\mathcal{X}$. Since

$$
\begin{aligned}
& d^{*}=(-1)^{q} *^{-1} d *, \\
& a^{*}=(-1)^{q-1} *^{-1} a *
\end{aligned}
$$

holds on $q$-forms, where by $*$ is meant the Hodge star operator related to a Riemannian metric on $\mathcal{X}$, the system for $a$ reduces to $\left[d, *^{-1} a *\right]+\left[*^{-1} d *, a\right]=0$ up to the inessential multiple $(-1)^{q-1}$ on $q$-forms. It is linear over $\mathbb{R}$ but fails to be so over $\mathbb{C}$.

To see if the system possesses solutions $a$ among nonclosed one-forms $a$, we consider it in a local chart $U$ on $\mathcal{X}$ with coordinates $x=\left(x^{1}, \ldots, x^{n}\right)$. In these coordinates the form $a$ can be written as $a=a_{1} d x^{1}+\ldots+a_{n} d x^{n}$, where $a_{1}, \ldots, a_{n}$ are smooth functions of $x$. Moreover, any bundle $\Lambda^{q}$ is trivial over $U$ under the representation of a form

$$
u(x)=\sum_{\substack{I=\left(i_{1}, \ldots, i_{q}\right) \\ 1 \leqslant i_{1}<\ldots<i_{q} \leqslant n}} u_{I}(x) d x^{i_{1}} \wedge \ldots \wedge d x^{x_{q}}
$$

by the $k_{q}$-column $\left(u_{I}(x)\right)$ of its coefficients, $k_{q}$ being the binomial coefficient $C_{n}^{q}$. The operator $d+a$ on $q$-forms is represented by a $\left(k_{q+1} \times k_{q}\right)$-matrix of first order partial differential operators. On assuming the canonical metric on $\mathbb{C}^{k_{q}}$ we get the formula

$$
\Delta_{a}=\Delta_{0}-\sum_{j=1}^{n}\left(\left(a_{j}(x)-\overline{a_{j}(x)}\right) \frac{\partial}{\partial x^{j}}+\frac{1}{2} \frac{\partial}{\partial x^{j}}\left(a_{j}(x)-\overline{a_{j}(x)}\right)\right)+|a(x)|^{2}
$$

provided the coefficients of $a$ satisfy

$$
\frac{\partial}{\partial x^{k}} a_{j}+\frac{\partial}{\partial x^{j}} \bar{a}_{k}=0
$$

for all $1 \leqslant j \leqslant k \leqslant n$.

Example 5.1. For $i=1, \ldots, n$, choose $a_{i}(x)=a_{i, 1} x^{1}+\ldots+a_{i, n} x^{n}+c_{i}$, where $A=\left(a_{i, j}\right)$ is an $(n \times n)$-matrix of complex numbers and $c_{i}$ complex numbers independent of $x$. Then system (5.1) is fulfilled if and only if $a_{j, k}+\bar{a}_{k, j}=0$ for all $1 \leqslant j \leqslant k \leqslant n$, i.e., the matrix $A$ is skew-Hermitean. An easy calculation shows that

$$
d a=-\sum_{1 \leqslant i<j \leqslant n} \frac{\partial}{\partial x^{j}}\left(a_{i}(x)+\overline{a_{i}(x)}\right) d x^{i} \wedge d x^{j}=-\sum_{1 \leqslant i<j \leqslant n}\left(a_{i, j}+\bar{a}_{i, j}\right) d x^{i} \wedge d x^{j},
$$

which need not vanish.

Under conditions (5.1), if moreover the differential form $a$ is real valued, then the Laplacian $\Delta_{a}$ reduces to $\Delta_{0}+|a(x)|^{2}$.

\section{Analytic torsion}

For elliptic complexes on compact manifolds the cohomology is represented by harmonic sections, i.e., those belonging to the null spaces of Laplacians. Hence, the harmonic spaces might substitute for the cohomology of Fredholm quasicomplexes, provided the Hodge theory holds. For manifolds with boundary the Hodge theory reduces to the Neumann problem after Spencer, and so the question arises if it is elliptic. 
The calculations of Section 2 show that the Laplace operators under the homogeneous Neumann conditions actually remain the only efficient tool to reveal resolving properties of an arbitrary sequence of differential operators on a manifold with boundary. The null spaces of the Laplace operators may substitute for the cohomology of such a sequence while their dimensions can be thought of as generalised Betti numbers. This agrees completely with the definition of the Euler characteristics for quasicomplexes of Banach spaces given in [11]. Moreover, the Fredholm property of the Neumann problem after Spencer allows one to introduce the concept of analytic torsion for a sequence. If it is independent on the choice of Hermitean structure of the sequence and thus reveals a topological nature should be a subject of special treatments. In this section we outline the concept of analytic torsion following to [8].

The Reidemeister torsion is a global invariant of a cell decomposition of a manifold and of an acyclic representation of its fundamental group. It is an invariant of the piecewise linear structure of the manifold. The Reidemeister torsion for an arbitrary finite-dimensional unimodular representation of the fundamental group can be defined as a canonical norm on the determinant line of the cohomology of a manifold. It is a multiplicative analogue of the Euler characteristic in the case of manifolds of odd dimension. (The Euler characteristic of a closed manifold is trivial in the odd-dimensional case.) Formulas for the Reidemeister torsion of the Cartesian product of two manifolds are similar to the multiplicative property of the Euler characteristic. The analytic torsion was introduced in [8] for a closed Riemannian manifold $\{\mathcal{X}, g\}$ with an acyclic orthogonal representation of the fundamental group $\pi_{1}(\mathcal{X})$. It is equal to the product of the corresponding powers of the determinants of the Laplace operators on differential forms of $\Omega \cdot(\mathcal{X})$. These determinants are regularised with the help of the zeta-functions of the Laplacians. (The Reidemeister torsion can also be written by analogous formula where the Riemannian Laplacians are replaced by the combinatorial ones.) The analytic torsion is defined with the help of a Riemannian metric $g$ on $\mathcal{X}$. However, it is independent of $g$ in the acyclic case, see [8]. So it is an invariant of the smooth structure on $\mathcal{X}$ and behaves in much the same way as the Reidemeister torsion. As was conjectured in [8], for any compact closed manifold $\mathcal{X}$ and acyclic representation $\rho$ of the fundamental group $\pi_{1}(\mathcal{X})$ the Reidemeister torsion of $\{\mathcal{X}, \rho\}$ has proven to be equal to the analytic torsion of $\{\mathcal{X}, \rho\}$.

On returning to sequence (0.1) we observe that it coicides with the de Rham complex up lower order operators. Hence, in the algebra of boundary value problems on $\mathcal{X}$ the sequence bears the same principal symbol structure as the de Rham complex. In particular, the tangential and normal components of a differential form $u \in \Omega^{i}(\mathcal{X})$ on the boundary of $\mathcal{X}$ with respect to sequence (0.1) coincide with those with respect to the de Rham complex. They are denoted by $t(u)$ and $n(u)$, respectively, so that $u=t(u)+d \varrho \wedge n(u)$ on $\partial \mathcal{X}$ where $\varrho$ is a defining function of the boundary with $|d \varrho|=1$ on $\partial \mathcal{X}$, see Section 3.2.2 in [10]. The Neumann problem after Spencer for sequence $(0.1)$ at step $i$ consists in finding, given any $f \in \Omega^{i}(\mathcal{X})$, a form $u \in \Omega^{i}(\mathcal{X})$ satisfying

$$
\begin{aligned}
\Delta_{a} u & =f \text { in } \mathcal{X}, \\
n(u) & =0 \text { on } \partial \mathcal{X}, \\
n((d+a) u) & =0 \text { on } \partial \mathcal{X} .
\end{aligned}
$$

By the above, the Laplace operator $\Delta_{a}^{i}$ is elliptic in $\mathcal{X}$. The boundary conditions are coercive for the Laplacian, see e.g. [5]. Thus, (6.1) is a classical elliptic boundary value problem in $\mathcal{X}$. Moreover, it is formally selfadjoint with respect to the Green formula.

Elliptic theory applies well to problem (6.1). All solutions $u \in H^{2}\left(\mathcal{X}, \Lambda^{i}\right)$ of the homogeneous problem corresponding to (6.1) belong actually to the space $\Omega^{i}(\mathcal{X})$, and they form a finite dimensional space $\mathcal{H}_{a}^{i}(\mathcal{X})$. The elements of $\mathcal{H}_{a}^{i}(\mathcal{X})$ are called harmonic forms. Denote by $H_{a}$ the orthogonal projection of $L^{2}\left(\mathcal{X}, \Lambda^{i}\right)$ onto $\mathcal{H}_{a}^{i}(\mathcal{X})$. Given any $f \in L^{2}\left(\mathcal{X}, \Lambda^{i}\right)$, the equation $\Delta_{a} u=f-H_{a} f$ has a unique solution $u$ in $H^{2}\left(\mathcal{X}, \Lambda^{i}\right)$ orthogonal to $\mathcal{H}_{a}^{i}(\mathcal{X})$. The operator $f \mapsto G_{a} f:=u$ is called the Green (or Neumann after Spencer) operator and it maps $L^{2}\left(\mathcal{X}, \Lambda^{i}\right)$ 
continuously into $H^{2}\left(\mathcal{X}, \Lambda^{i}\right)$. The Green operator is known to be a selfadjoint operator in $L^{2}\left(\mathcal{X}, \Lambda^{i}\right)$. Its smoothness properties are deduced from the fact that $G_{a}$ is an operator of order -2 in the algebra of boundary value problems on $\mathcal{X}$ outlined in [3]. By the above,

$$
f=H_{a} f+P_{a}(d+a) f+(d+a) P_{a} f+(d+a)^{*}\left[d+a, G_{a}\right] f
$$

for all $f \in L^{2}\left(\mathcal{X}, \Lambda^{i}\right)$, where $P_{a}=(d+a)^{*} G_{a}$. The commutator $\left[G_{a}, d+a\right]$ need not vanish unless $a$ is a closed one-form.

Lemma 6.1. Suppose that the perturbation satisfies $n(d a)=0$ on the boundary of $\mathcal{X}$. Then

$$
\left[d+a, G_{a}\right]=G_{a}\left[(d+a)^{*}, d a\right] G_{a} .
$$

Proof. Denote by $\mathcal{N}_{a}^{i}(\mathcal{X})$ the subspace of $\Omega^{i}(\mathcal{X})$ consisting of those differential forms $u$ which satisfy the boundary conditions in (6.1), i.e., $n(u)=0$ and $n((d+a) u)=0$ on $\partial \mathcal{X}$.

We first show that $A=d+a$ maps $\mathcal{N}_{a}^{i}(\mathcal{X})$ continuously into $\mathcal{N}_{a}^{i+1}(\mathcal{X})$. If $u \in \mathcal{N}_{a}^{i}(\mathcal{X})$, then $n(A u)=0$ and it remains to check if $n\left(A^{2} u\right)=0$ holds. We get $A^{2} u=d a \wedge u$ and

$$
\begin{aligned}
d a \wedge u & =(t(d a)+d \varrho \wedge n(d a)) \wedge(t(u)+d \varrho \wedge n(u))= \\
& =t(d a) \wedge t(u)+d \varrho \wedge(t(d a) \wedge n(u)+n(d a) \wedge t(u))
\end{aligned}
$$

on $\partial \mathcal{X}$ whence $n(d a \wedge u)=0$, as desired.

On applying the operator $A$ to the equality $I=H_{a}+\Delta_{a} G_{a}$ on $\Omega^{i}(\mathcal{X})$ from the left and from the right we see that $A \Delta_{a} G_{a}=\Delta_{a} G_{a} A$, for both $A H_{a}$ and $H_{a} A$ vanish. Thus,

$$
\Delta_{a}\left[A, G_{a}\right]=\left[\Delta_{a}, A\right] G_{a}
$$

and so the equality $G_{a} \Delta_{a}\left[A, G_{a}\right] u=G_{a}\left[\Delta_{a}, A\right] G_{a} u$ holds for all $u \in \Omega^{i}(\mathcal{X})$.

From the construction of the Green operator we deduce that $\left(G_{a} \Delta_{a}\right) g=g-H_{a} g$ for all $g \in \mathcal{N}_{a}^{i+1}(\mathcal{X})$. Now, if $u \in \Omega^{i}(\mathcal{X})$ then $g=\left[A, G_{a}\right] u=A\left(G_{a} u\right)-G_{a}(A u)$ belongs to the space $\mathcal{N}_{a}^{i+1}(\mathcal{X})$, for $A$ maps $\mathcal{N}_{a}^{i}(\mathcal{X})$ into $\mathcal{N}_{a}^{i+1}(\mathcal{X})$. Hence it follows that

$$
G_{a} \Delta_{a}\left[A, G_{a}\right]=\left(I-H_{a}\right)\left[A, G_{a}\right]
$$

is valid on all of $\Omega^{i}(\mathcal{X})$. As $H_{a} A=0$ and $H_{a} G_{a}=0$, we get $G_{a} \Delta_{a}\left[A, G_{a}\right]=\left[A, G_{a}\right]$, on the one hand.

On the other hand, an easy calculation gives

$$
\begin{aligned}
{\left[\Delta_{a}, A\right] } & =\left(A^{*} A+A A^{*}\right) A-A\left(A^{*} A+A A^{*}\right)= \\
& =A^{*} A^{2}-A^{2} A^{*}= \\
& =\left[A^{*}, A^{2}\right]
\end{aligned}
$$

showing (6.2).

From Lemma (6.1) it follows that $P_{a}(d+a) f+(d+a) P_{a} f=f-K_{a} f$ for all $f \in \Omega^{i}(\mathcal{X})$, where $K_{a}=H_{a}+A^{*} G_{a}\left[A^{*}, d a\right] G_{a}$ is a pseudodifferential operator of order -2 on $\mathcal{X}$. Hence, $P_{a}$ is a parametrix of sequence (4.1) whose remainder $K_{a}$ is "smaller" than the remainder of the parametrix constructed in Theorem 4.2. It is to be expected that the standard procedure using the formal Neumann series for $\left(I-K_{a}\right)^{-1}$ works to construct a parametrix modulo smoothing operators but we will not develop this point here.

Given any nonnegative linear mapping $L$ of a unitary space $V$, one defines the zeta function of $L$ by $\zeta_{L}(s)=\operatorname{tr} L^{-s}$, where tr stands for the functional trace. Thus, we get

$$
\zeta_{L}(s)=\sum_{j} \lambda_{j}^{-s},
$$


where the sum is over all nonzero eigenvalues of $L$. For selfadjoint mappings $L$ determined by elliptic operators or boundary value problems, it is known that the eigenvalues of $L$ can be arranged in a monotone increasing sequence converging to infinity. Moreover, $\zeta_{L}(s)$ has a meromorphic extension to the entire complex plane which is regular at $s=0$. On formally differentiating the series term-by-term one obtains

$$
\zeta_{L}^{\prime}(s)=-\sum_{j} \frac{\log \lambda_{j}}{\lambda_{j}^{s}}
$$

and so if the functional determinant is well defined and different from zero, then it should be given by $\operatorname{det} L=\exp \left(-\zeta_{L}^{\prime}(0)\right)$. Since the analytic continuation of the zeta function is regular at zero, this can be rigorously adopted as a definition of the determinant.

For a sequence

$$
\left(H^{\cdot}, d\right): 0 \longrightarrow H^{0} \stackrel{d^{0}}{\longrightarrow} H^{1} \stackrel{d^{1}}{\longrightarrow} \ldots \stackrel{d^{N-1}}{\longrightarrow} H^{N} \longrightarrow 0
$$

of unitary spaces, the analytic torsion (or determinant) is now introduced by the formula

$$
\begin{aligned}
\log T\left(H^{\cdot}, d\right) & =\frac{1}{2} \sum_{i=0}^{N}(-1)^{i+1} i \log \operatorname{det} \Delta^{i}= \\
& =\sum_{i=0}^{N} \log \left(\operatorname{det} \Delta^{i}\right)^{(-1)^{i+1} i / 2}= \\
& =\log \prod_{i=0}^{N}\left(\operatorname{det} \Delta^{i}\right)^{(-1)^{i+1} i / 2}
\end{aligned}
$$

or

$$
T\left(H^{*}, d\right)=\prod_{i=0}^{N}\left(\operatorname{det} \Delta^{i}\right)^{(-1)^{i+1} i / 2},
$$

where $\Delta^{i}=d^{i-1} d^{i-1 *}+d^{i *} d^{i}$ are the Laplacians of the sequence $\left(H^{*}, d\right)$. It is easy to check that for short sequences (i.e, $N=1$ ) the analytic torsion reduces to $\left|\operatorname{det} d^{0}\right|$.

Example 6.2. Consider the sequence of symbols corresponding to perturbation (0.1). It is the complex

$$
\left(\Lambda T_{x}^{*} \mathcal{X}, \sigma(\xi)\right): 0 \longrightarrow \Lambda^{0} T_{x}^{*} \mathcal{X} \stackrel{\sigma(\xi)}{\longrightarrow} \Lambda^{1} T_{x}^{*} \mathcal{X} \stackrel{\sigma(\xi)}{\longrightarrow} \ldots \stackrel{\sigma(\xi)}{\longrightarrow} \Lambda^{n} T_{x}^{*} \mathcal{X} \longrightarrow 0
$$

parametrised by a point $x \in \mathcal{X}$ and a vector $\xi \in T_{x}^{*} \mathcal{X}$ different from zero, where $\sigma(\xi) v=\xi \wedge v$ for all $v \in \Lambda^{i} T_{x}^{*} \mathcal{X}$. Choosing an orthonormal basis in each tangent space $T_{x} \mathcal{X}$, we endow the spaces $\Lambda^{i} T_{x}^{*} \mathcal{X}$ with a unitary structure in the usual manner. The dimension of $\Lambda^{i} T_{x}^{*} \mathcal{X}$ is equal to the binomial coefficient $k_{i}:=C_{n}^{i}$. The Laplacians of the complex $\left(\Lambda^{*} T_{x}^{*} \mathcal{X}, \sigma(\xi)\right)$ reduce to $\Delta^{i}=|\xi|^{2} I^{i}$, where $I^{i}$ is the identity mapping of $\Lambda^{i} T_{x}^{*} \mathcal{X}$. Hence it follows that $\operatorname{det} \Delta^{i}=|\xi|^{2 k_{i}}$, and so the analytic torsion is

$$
\begin{aligned}
T\left(\Lambda \cdot T_{x}^{*} \mathcal{X}, \sigma(\xi)\right) & =\prod_{i=0}^{n}\left(|\xi|^{2 k_{i}}\right)^{(-1)^{i+1} i / 2}= \\
& =|\xi|^{-0 k_{0}+1 k_{1}-\ldots+(-1)^{n-1} n k_{n}} .
\end{aligned}
$$

If $n=1$, then the analytic torsion just amounts to $|\xi|$. For all $n>1$, it reduces to 1 since the exponent is $n(1-1)^{n-1}$ by the binomial formula.

Formula (6.3) can be used to introduce the concept of analytic torsion for quasicomplex (0.1). To this end, as $\Delta^{i}$ one takes the Laplacians $\Delta_{a}^{i}$ of (0.1) under the homogeneous Neumann 
(after Spencer) boundary conditions, cf. (6.1). Of course, the product of the eigenvalues of these Laplacians is infinite, so the notion of determinant must be regularised by means of $\operatorname{det} \Delta_{a}^{i}=\exp \left(-\zeta_{\Delta_{a}}^{\prime}(0)\right)$. If defined in this way, the analytic torsion is a subtle spectral invariant. It is easy to check that the zeta function for the Laplacian on the simplest manifold with boundary, the interval, is a slight variation of the Riemann zeta function. Geometers can perhaps study the dependence of analytic torsion on the particular choice of Riemannian metric on $\mathcal{X}$, a technique usually unavailable to number theorists due to rigidity results.

The first author gratefully acknowledges the financial support of the Islamic Center Association for Guidance and Higher Education.

\section{References}

[1] S.Agmon, A.Douglis, L.Nirenberg, Estimates near the boundary for solutions of elliptic partial differential equations satisfying general boundary conditions. Pt. I, Comm. Pure Appl. Math., 12(1959), 623-727.

[2] C.-G.Ambrozie, F.-H.Vasilescu, Banach Space Complexes, Mathematics and its Applications, Vol. 334, Kluwer Academic Publishers, Dordrecht, NL, 1995.

[3] L.Boutet de Monvel, Boundary problems for pseudodifferential operators, Acta Math., $\mathbf{1 2 6}(1971), 11-51$.

[4] L.Hörmander, $L^{2}$ estimates and existence theorems for the $\bar{\partial}$ operator, Acta Math., 113(1965), no. 1-2, 89-152.

[5] I.Malass, N.Tarkhanov, J. Siber. Fed. Univ., Math. and Phys., 12(2019), no. 4, 455-465. DOI: 10.17516/1997-1397-2019-12-4-455-465

[6] S.P.Novikov, On Metric-Independent Exotic Homology, Proc. Steklov Inst. Math., 251(2005), 206-212.

[7] M.Putinar, Some invariants for semi- Fredholm systems of essentially commuting operators, J. Operator Theory, 8(1982), 65-90.

[8] D.B.Ray, I.M.Singer, Reidemeister torsion and the Laplacian on Riemannian manifolds, Adv. in Math., 7(1971), 145-210.

[9] D.C.Spencer, Harmonic integrals and Neumann problems associated with linear partial differential equations, In: Outlines of Joint Soviet-American Symposium on Partial Differential Equations, Novosibirsk, 1963, 253-260.

[10] N.Tarkhanov, Complexes of Differential Operators, Kluwer Academic Publishers, Dordrecht, NL, 1995.

[11] N.Tarkhanov, Euler characteristic of Fredholm quasicomplexes, Funct. Anal. and its Appl., 41(2007),318-322.

[12] K.Krupchyk, N.Tarkhanov, J.Tuomela, Elliptic quasicomplexes in Boutet de Monvel algebra, J. of Funct. Anal., 247(2007), 202-230.

[13] D.Wallenta, A Lefschetz fixed point formula for elliptic quasicomplexes, Integr. Equ. Oper. Theory, 78(2014), no. 4, 577-587.

[14] R.O.Wells, Differential Analysis on Complex Manifolds, Springer-Verlag, New York, 1980. 
[15] E.Witten, Supersymmetry and Morse theory, J. Diff. Geom., 17(1982), 661-692.

\title{
Возмущение комплекса де Рама
}

Исан Малас

Николай Тарханов

Потсдамский университет

Потсдам, Германия

\begin{abstract}
Аннотация. Рассмотрим возмущение комплекса де Рама на компактном многообразии с краем. Это возмущение выходит за рамки комплексов, и поэтому когомологии к нему не относятся. С другой стороны, его кривизна "мала", поэтому существует естественный способ ввести характеристику Эйлера и разработать теорию Лефшеца для возмущения. Данная работа предназначена для попытки разработать теорию когомологий для произвольных последовательностей линейных отображений.
\end{abstract}

Ключевые слова: комплекс де Рама, когомологии, теория Ходжа, проблема Неймана. 